\title{
ELETROTERAPIA APLICADA AO \\ MEMBRO SUPERIOR ESPÁSTICO \\ DE PACIENTES COM ACIDENTE \\ VASCULAR CEREBRAL - UM ESTUDO DE REVISÃO
}

Camila Barreto*, Haline Carteado*, Louise Raissa Viégas*, Maria Carolina Bastos*, Murilo Novais*, Daniel Dominguez*

Endereço para correspondência: Camila da Silva Barreto - kammylllla@hotmail.com

* Universidade Federal da Bahia

\begin{abstract}
Resumo
Introdução: A espasticidade é uma alteração do tônus muscular secundaria a uma lesão do neurônio motor superior geralmente decorrente de um distúrbio vascular cerebral. A estimulação elétrica neuromuscular vem sendo utilizada pelos fisioterapeutas com o objetivo de reduzir a espasticidade, melhorar a capacidade motora e o desempenho na realização das atividades de vida diária. Objetivo: Apresentar os efeitos da aplicação de estímulos elétricos neuromusculares sobre os músculos espásticos do membro superior de pacientes com sequelas de Acidente Vascular Cerebral (AVC). Metodologia: O estudo utilizou o protocolo de orientações do PRISMA para a elaboração da revisão sistemática. A busca bibliográfica foi realizada no período entre dezembro de 2014 e março de 2015 nas bases de dados: MedLine/PubMed, PEDro, LILACS e Scielo. Foram utilizados os seguintes descritores: Espasticidade, Muscle Spasticity, Fisioterapia e Physical Therapy Modalities. Resultados: Foram selecionados 6 estudos. As pesquisas mostram uma redução significativa da espasticidade em músculos do membro superior espástico de pacientes com sequelas de AVC, tratados com eletroestimulação muscular. Outros resultados também foram encontrados, como a redução da dor e da contratura muscular, a melhora da função motora dos membros superiores e o aumento da amplitude de movimento ativa de punho e dedos. Não existe consenso entre os parâmetros de aplicação do tratamento com eletroterapia. Conclusão: Os estudos mostram que a eletroterapia, aplicada ao membro superior espástico do paciente com sequelas de AVC, parece contribuir para a redução da espasticidade, melhora das complicações associadas e incremento da capacidade funcional. Porém, ainda é baixa a qualidade metodológica dos estudos e reduzido o número de artigos sobre o tema.

Palavras-chave: Espasticidade Muscular; Modalidades de Fisioterapia; Acidente Vascular Cerebral; Terapia por Estimulação Elétrica.
\end{abstract}




\title{
ELECTROTHERAPY APPLIED TO SPASTIC UPPER LIMB ON PATIENTS WITH CEREBROVASCULAR ACCIDENT
}

\begin{abstract}
Introduction: Spasticity is a change in muscle tone secondary to an upper motor neuron injury usually due to a brain vascular disorder. Neuromuscular electrical stimulation has been used by physiotherapists in order to reduce spasticity, improve motor skills and performance in activities of daily living. Objective: To present the effects of applying neuromuscular electrical stimulation of the upper limb spastic muscles of patients with stroke sequelae. Methodology: The study used the PRISMA guidelines protocol for the preparation of a systematic review. The literature search was conducted in the period between December 2014 and March 2015 in the databases: MEDLINE / PubMed, PEDro, LILACS and Scielo. The following parameters were used: Spasticity, Muscle Spasticity, Physical Therapy and Physical Therapy Modalities. Results: A total of 6 studies. Researches show a significant reduction of spasticity in spastic upper limb muscles of patients with stroke sequelae, treated with muscle electrostimulation. Other results were also found, such as reducing pain and muscle spasm, improvement of motor function of the upper and increased active range of motion of the wrist and fingers. There is no consensus between the application parameters of treatment with electrotherapy. Conclusion: These studies show that the electrical stimulation applied to the upper limb spasticity patients with stroke, seems to contribute to the reduction of spasticity, improvement of associated complications and functional capacity. However, it is still low methodological quality and reduced the number of studies on the subject.
\end{abstract}

Keywords: Muscle Spasticity; Physical Therapy Modalities; Stroke; Electric Stimulation Therapy.

\section{INTRODUÇÃO}

O distúrbio na circulação cerebral, desencadeado pelo AVC, provoca insuficiência do sistema nervoso central e se manifesta inicialmente através de um déficit neurológico agudo, responsável pela redução da função com duração mínima de 24 horas. (1).

Pesquisas apontam que $75 \%$ dos sobreviventes evoluem com algum tipo de deficiência, sendo as extremidades superiores as áreas mais acometidas. ${ }^{(1)}$ Aproximadamente $30-66 \%$ dos indivíduos acometidos não conseguem utilizar o membro superior afetado após a lesão, provocando grande impacto na realização das atividades da vida diária. (2) O déficit de movimento pode alterar tanto o controle motor global da extremidade superior como a destreza manual, devido à perda sensorial e às deficiências na integração sensório-motora. ${ }^{(3)}$

A espasticidade é uma das manifestações clínicas do AVC, resultante da lesão do neurônio motor superior no sistema nervoso central. (4) Esta manifestação clínica pode ser definida como uma desordem motora caracterizada por uma hiper-reflexia velocidade dependente. ${ }^{(4)} \mathrm{A}$ espasticidade severa reduz a flexibilidade, ocasiona erros de postu- 
ra e reduz a mobilidade funcional além de provocar dor articular, contratura e dificuldade de posicionamento para o conforto e a higiene. ${ }^{(5)}$

Existe uma diversidade de intervenções terapêuticas que pode auxiliar à redução da espasticidade de acordo a necessidade clínica e a probabilidade de sucesso para alcançar objetivos específicos. ${ }^{(5)}$ Como tratamento médico pode-se incluir os medicamentos antispásticos como baclofeno, diazepam, tizanidina, ou dantroleno e a toxina botulínica, utilizada para relaxar um grupo muscular específico. ${ }^{(5)}$

Entre os procedimentos fisioterapêuticos encontram-se o alongamento muscular, os exercícios de amplitude de movimento que ajudam a prevenir o encurtamento muscular, a atrofia e a progressão da gravidade dos sintomas. ${ }^{(6)}$ A estimulação elétrica neuromuscular também vem sendo utilizada pelos fisioterapeutas com o objetivo de reduzir a espasticidade. Estudos clínicos têm demonstrado que a estimulação elétrica neuromuscular pode melhorar a função motora e o desempenho para realizar as atividades de vida diária. $(4,6,7,8)$

Assim, esta revisão tem como objetivo apresentar os resultados de estudos sobre os efeitos da eletroestimulação muscular em músculos espásticos do membro superior de pacientes com sequelas de AVC.

\section{MÉTODOS}

Para a elaboração desta revisão sistemática foi utilizado o protocolo de orientações do PRISMA (Preferred Reporting Items for Systematic Reviews and Meta-Analyses). Apenas foram selecionados estudos encontrados nas seguintes bases de dados: Medical Literature Analysis and Retrieval System Online (MedLine/PubMed), Physiotherapy Evidence Database (PEDro), Literatura Latino-Americana e do Caribe em Ciências da Saúde (LILACS) e Scielo. $\mathrm{Na}$ busca bibliográfica, realizada no período entre dezembro de 2014 e março de 2015, foram utilizados os seguintes descritores: Espasticidade,
Muscle Spasticity, Fisioterapia e Physical Therapy Modalities.

Na base de dados MedLine/PubMed, a busca bibliográfica foi realizada através dos MeSH (Muscle Spasticity e Physical Therapy Modalities), sendo usada a seguinte fórmula: ("Muscle Spasticity"[Mesh]) AND "Physical Therapy Modalities"[Mesh].

Como critério de inclusão define-se que apenas foram selecionados ensaios clínicos controlados e randomizados publicados nos últimos dez anos, que tenham utilizado a eletroestimulação neuromuscular para tratar a espasticidade do membro superior de indivíduos com sequelas de AVC. Como critérios de exclusão, foram excluídos artigos de revisão, meta-análises e editorial, assim como, estudos publicados em formato de protocolo de ensaio clínico por não possuírem dados finais para serem analisados nesta revisão sistemática.

Foram encontrados um total de 90 artigos na base de dados MedLine/PubMed, 3 na PEDro, 31 na LILACS e 13 na Scielo. A seleção dos artigos foi realizada por todos os autores simultaneamente, inicialmente através da leitura dos títulos. Os casos de divergência de opiniões dos autores sobre a possível seleção dos artigos, foram discutidos através da leitura do resumo. Para finalizar o processo de seleção, foi realizada a leitura na íntegra de todos os artigos pré-selecionados.

Foram selecionados 6 artigos e foi realizada uma análise qualitativa de acordo com os objetivos do estudo.

\section{RESULTADOS}

Nessa revisão sistemática, foram analisados 6 artigos completos que atenderam aos critérios de inclusão e exclusão pré-determinados. Os resultados de cada artigo foram sumarizados e expostos na Tabela 1. 
Tabela 1 - Dados dos estudos selecionados

\begin{tabular}{|c|c|c|c|c|}
\hline AUTORES & AMOSTRA $(\mathrm{N})$ & ELETROESTIMULAÇÃO & REABILITAÇÃO & DESFECHO \\
\hline $\begin{array}{l}\text { Wang BH } \\
\text { Lin CL } \\
(2 \mathrm{O} 14)\end{array}$ & $\begin{array}{c}15 \\
\text { Indivíduos com } \\
\text { mais de } 6 \text { meses } \\
\text { após o AVC }\end{array}$ & $\begin{array}{l}\text { Eletroacupuntura (12 } \\
\text { sessões, } 2 \text { x/semana) }\end{array}$ & $\begin{array}{c}\text { Exercícios passivos, } \\
\text { alongamentos, } \\
\text { fortalecimento, } \\
\text { treino de equilíbrio }\end{array}$ & $\begin{array}{l}\text { Redução significativa } \\
\text { apenas dos valores de } \\
\text { R1 e R2 do cotovelo }\end{array}$ \\
\hline $\begin{array}{l}\text { Malhotra S } \\
\text { Rosewilliam S } \\
\qquad(2 \mathrm{O} 12)\end{array}$ & $\begin{array}{l}90 \\
\text { Indivíduos a } \\
\text { partir da } 6^{a} \\
\text { semana do } \\
\text { primeiro AVC }\end{array}$ & $\begin{array}{c}\text { Estimulação elétrica } \\
\text { neuromuscular de } \\
\text { superfície ( } 2 x / \text { dia, } 5 \\
\text { dias/semana, durante } 6 \\
\text { semanas) }\end{array}$ & $\begin{array}{c}\text { Exercícios passivos } \\
\text { e ativo assistido, } \\
\text { funcional }\end{array}$ & $\begin{array}{l}\text { Redução da dor e } \\
\text { da contratura. Não } \\
\text { há efeito de longo } \\
\text { prazo na redução da } \\
\text { espasticidade }\end{array}$ \\
\hline $\begin{array}{l}\text { Mukherjee M } \\
\text { McPeak LK } \\
\text { (2007) }\end{array}$ & $\begin{array}{c}7 \\
\text { Indivíduos a } \\
\text { partir do } 3^{\circ} \text { mês } \\
\text { do primeiro AVC }\end{array}$ & $\begin{array}{c}\text { Eletroacupuntura }(2 x / \\
\text { semana eletroestimulação } \\
\text { e fisioterapia por } 12 \\
\text { semanas) }\end{array}$ & $\begin{array}{c}\text { Exercícios de } \\
\text { fortalecimento } \\
\text { (isométricos e ativo } \\
\text { assistido) }\end{array}$ & $\begin{array}{l}\text { Redução da } \\
\text { espasticidade }\end{array}$ \\
\hline $\begin{array}{l}\operatorname{Lin} Z \\
\text { Yan T } \\
(2011)\end{array}$ & $\begin{array}{l}\quad 37 \\
\text { Indivíduos nos } \\
\text { três primeiros } \\
\text { meses após o } \\
\text { AVC }\end{array}$ & $\begin{array}{l}\text { Estimulação elétrica } \\
\text { neuromuscular por } 5 \text { dias, } \\
\text { durante } 3 \text { semanas ( } 30 \\
\text { minutos de aplicação) }\end{array}$ & $\begin{array}{l}\text { Fisioterapia } \\
\text { Convencional } \\
\text { e Terapia } \\
\text { Ocupacional não } \\
\text { detalhada }\end{array}$ & $\begin{array}{l}\text { Melhora significativa } \\
\text { da função motora dos } \\
\text { membros superiores } \\
\text { afetados, os efeitos } \\
\text { persistem por pelo } \\
\text { menos } 6 \text { meses } \\
\text { após interromper o } \\
\text { tratamento }\end{array}$ \\
\hline $\begin{array}{l}\text { Hara Y et al. } \\
\qquad(2006)\end{array}$ & $\begin{array}{l}16 \\
\text { Indivíduos com } \\
\text { mais de } 1 \text { ano } \\
\text { após o AVC }\end{array}$ & $\begin{array}{c}\text { Estimulação elétrica } \\
\text { funcional ( } 2 x \text { por semana } \\
\text { durante } 4 \text { meses) }\end{array}$ & $\begin{array}{c}\text { Terapia } \\
\text { Ocupacional } \\
\text { Convencional }\end{array}$ & $\begin{array}{l}\text { Melhora da amplitude } \\
\text { de movimento ativa de } \\
\text { punho e dedos para } \\
\text { extensão. }\end{array}$ \\
\hline $\begin{array}{l}\text { Liu W et al. } \\
\text { (2008) }\end{array}$ & $\begin{array}{l}10 \\
\text { Indivíduos com } \\
\text { mais de } 2 \text { anos } \\
\text { após o AVC }\end{array}$ & $\begin{array}{l}\text { Eletroacupuntura }(2 x \\
\text { por semana durante } 6 \\
\text { semanas) }\end{array}$ & $\begin{array}{l}\text { Exercícios de } \\
\text { fortalecimento }\end{array}$ & $\begin{array}{l}\text { Redução significativa } \\
\text { da espasticidade e } \\
\text { recuperação motora } \\
\text { funcional. }\end{array}$ \\
\hline
\end{tabular}

\section{DISCUSSÃO}

De acordo aos estudos, a estimulação elétrica muscular aplicada aos músculos espásticos do membro superior parece melhorar significativamente a função física e a capacidade funcional de pacientes com sequelas de AVC.
Para avaliar o grau da espasticidade no membro superior acometido, todos os estudos optaram por utilizar a escala de Ashworth. ${ }^{(9-14)}$ Apenas dois artigos usaram a goniometria para avaliar a amplitude de movimento ${ }^{(10,11)}$ e a escala Fugh Meyer para verificar a motricidade do membro superior tratado. $(9,10)$ Apenas um autor avaliou a capacidade funcional para realizar as atividades básicas da vida diária, 
através do Índice de Barthel Modificado(9) e o dinamômetro para verificar o nível de força muscular. ${ }^{(10)}$

Os tratamentos de Fisioterapia Convencional do membro superior espástico para comparação com a eletroestimulação foram diversos, sendo a cinesioterapia passiva, cinesioterapia ativo-assistida, exercícios funcionais e de fortalecimento, os mais utilizados. Também no tratamento com a utilização da eletroestimulação muscular não houve um consenso. A aplicação da corrente elétrica ocorreu em diferentes grupos musculares, entre eles o supra espinhal, deltoide, extensores do punho, pronador redondo, pronador quadrado, flexor superficial dos dedos, flexor longo do polegar e braquiorradial. O período total de aplicação dos procedimentos terapêuticos variava entre 1 a 4 meses, e o tempo das sessões durava entre 30 a 40 minutos com uma frequência de 2 ou 3 vezes por semana.

Os parâmetros da corrente elétrica também eram distintos entre os estudos. A frequência da corrente utilizada variou de 2 a 50 hertz, o tempo on e off de 1 a 15 segundos e a largura de pulso foi de 30oms. Os estudos coincidiram em relação à intensidade da corrente, pois utilizaram a tolerância do paciente como parâmetro. Apenas um autor descreve ter aplicado a corrente elétrica a uma intensidade desagradável, porém tolerável, segundo a percepção individual do paciente. ${ }^{(2)}$

Segundo Cauraugh et al. (2000), (15) Peurala (2002) ${ }^{(16)}$ e Kimberley (2004), ${ }^{(17)}$ a paresia do membro superior é uma das deficiências funcionais primárias de pacientes com sequelas de AVC. A habilidade para a realização das atividades da vida diária é uma das metas mais importantes do processo de reabilitação. ${ }^{(18,19)} \mathrm{A}$ reabilitação, quando realizada precocemente, ajuda a melhorar de forma significativa a função motora nos membros superiores hemiplégicos dos pacientes, devido à ativação cortical proporcionada pela plasticidade neuronal.

Lin (2011), estudando a eficácia a longo prazo da estimulação neuromuscular em 18 pacientes encontrou resultados significativos na melhora da função motora do ombro e da mão no membro superior espástico. Em seu estudo foi observado que os efeitos positivos sobre a função motora do membro superior espástico, alcançados com a eletroestimulação muscular, continuaram após 3 a 6 meses após o tratamento. ${ }^{(9)}$

Já Malhotra (2013) apontou que a aplicação da estimulação neuromuscular no membro superior espástico resultou na redução da dor durante todo o estudo. Também observou efeitos benéficos na redução de contraturas musculares, embora não tenha encontrado resultados significativos sobre a redução da espasticidade. No estudo, o autor explica que a espasticidade e as contraturas musculares foram considerados fatores etiológicos do quadro álgico, e que a eficácia no tratamento dessas manifestações clínicas, seria, portanto, a redução da dor no membro superior espástico por sequelas de um AVC. ${ }^{(13)}$

O estudo de Hara (2006) também encontrou resultados positivos na utilização da eletroestimulação muscular como tratamento do membro superior espástico após um AVC. Ao analisar o trabalho múscular do antebraço hemiparético, verificou que a utilização da estimulação elétrica neuromuscular proporcionou uma melhora da amplitude de movimento ativa tanto de punho como de dedos. Foram avaliados os movimentos de extensão de punho e dedos com padrão espástico flexor e ao comparar com o desempenho motor do grupo controle, todos os pacientes tratados com a eletroestimulação muscular melhoraram a atividade motora da mão de forma significativa. ${ }^{(12)}$

Liu et al. (2008) e Wang et al. (2014) utilizaram a eletroacupuntura para tratar o membro superior espástico de pacientes com sequelas de AVC. Ao analisar os efeitos da eletroacupuntura na recuperação motora em dez sobreviventes de AVC, Liu et al. (2008) observaram que o tratamento conseguiu ajudar na redução da espasticidade. Mesmo com uma amostra reduzida, também observaram a presença de benefícios adicionais como a melhora da recuperação motora funcional quando combinado a exercícios de fortalecimento. ${ }^{(10)}$

Wang et al. (2014) também associaram o tratamento de eletroacupuntura com a Fisioterapia 
Convencional para a reabilitação do membro superior espástico após um AVC. Os seus resultados mostram um aumento significativo dos valores da amplitude de movimento passiva e do ângulo de reação muscular do cotovelo, indicando melhora no nível do componente dinâmico da espasticidade. Os efeitos sobre as medidas da articulação do punho não foram significativas entre o grupo submetido à Fisioterapia Convencional e os pacientes que realizaram a Fisioterapia Convencional associada à eletroacupuntura. Esses efeitos sobre o punho podem ser explicados, pois os pontos de acupuntura utilizados na aplicação do tratamento de eletroacupuntura estavam localizados próximos ao ventre muscular de grupos musculares relacionados principalmente ao movimento do cotovelo. ${ }^{(11)}$

Com resultados similares, Mukherjee (2007) também analisou o efeito da eletroacupuntura na espasticidade da articulação do punho em pacientes crônicos com sequelas de AVC. Em seus resultados não se observa uma redução significativa para o efeito imediato do tratamento de acupuntura. Porém, todos os indivíduos com exceção de um, mostraram uma redução da espasticidade do membro superior acometido ao finalizar o tratamento. Vale ressaltar, que o estudo apresentou problemas técnicos no instrumento de avaliação comprometendo seus resultados, pois os sinais eletromiográficos de dois participantes estavam incompletos. A atividade eletromiográfica para os músculos flexores de punho durante a extensão passiva da articulação não foi significativa quando são comparados os valores antes e após a eletroacupuntura. ${ }^{(14)}$

Em relação às limitações dos estudos é importante destacar o reduzido número amostral, a utilização de instrumentos de avaliação pouco precisos e objetivos e o fato de não terem verificado os efeitos a longo prazo do tratamento. $O$ uso da escala de Ashworth, pela maioria dos estudos, como principal instrumento para avaliar a espasticidade é outra limitação, visto que a validade e a precisão dessa escala não é suficiente para ser usada como uma medida da espasticidade. ${ }^{(20)}$

Por esses motivos e pela escassez de estudos sobre os efeitos da eletroestimulação no tratamen- to da espasticidade do membro superior de pacientes com sequelas de AVC, não é possível determinar conclusões significativas.

\section{CONCLUSÃO}

Os diferentes protocolos adotados pelos estudos tornaram difícil a comparação dos resultados. Porém, segundo os dados observados, a eletroterapia aplicada ao membro superior espástico do paciente com sequelas de AVC, associada ou não à Fisioterapia Convencional, parece contribuir para a redução da espasticidade e melhora de suas complicações.

Os resultados deste estudo de revisão sistemática sintetizam evidências sobre os efeitos da eletroterapia que podem contribuir para subsidiar as ações clínicas de profissionais que trabalham com esse perfil de paciente e que a utilizam, favorecendo a prática baseada em evidências.

Deve-se salientar que ainda são poucos os ensaios clínicos randomizados sobre o tema proposto. Assim, faz-se necessário a realização de novos estudos que investiguem o uso da eletroterapia utilizando métodos de avaliação mais precisos e que descrevam o método de tratamento possibilitando a elaboração de um consenso sobre os parâmetros da técnica.

\section{REFERÊNCIAS}

1. Yozbatiran N, Donmez B, Kayak N. Bozana $O$. Electrical stimulation of wrist and fingers for sensory and functional recovery in acute hemiplegia. Clin. rehab. 2006;20: 4-11.

2. Wang $C$, Li J, Zhao X, Wang Y, Wu D, Wang Y Stroke developmentin mainland China: past, present and future. Stroke. 2008;3:288-289.

3. Adkins-Muir DL, Jones TA. Cortical electrical stimulation combined with rehabilitative training: enhanced functional recovery and dendritic plasticity following focal cortical ischemia in rats. Neurol. res. 2003;25(8):780788. 
4. Sommerfeld DK, Eek EU, Svensson AK, Holmqvist LW, von Arbin MH. Spasticity after stroke: its occurrence and association with motor impairments and activity limitations. Stroke. 2004;35(1):134139.

5. Braddom RL. Physical Medicine and Rehabilitation. 4th ed. Philadelphia: Elsevier; 2010.

6. Kimberley TJ, Lewis SM, Auerbach EJ, Dorsey LL, Lojovich JM, Carey JR. et al. Electrical stimulation driving neuromuscular improvements and cortical in patients with stroke. Exp. brain. res. 2004;154:450-460.

7. Vuagnat $H$, Chantraine A. Shoulder pain in hemiplegia revisited:contribution of functional electricalstimulation and other therapies. J. rehab. med. 2003;35:49-56.

8. Knutson JS, Harley MY, Hisel TZ, Chae J. Improving hand function in stroke survivors: a pilot study of contralaterally controlled functional electric stimulation in chronic hemiplegia. Arch. phys. med. rehab. 2007;88:513-520.

9. Lin Z, Yan T. Long-term effectiveness of neuromuscular electrical stimulation for promoting motor recovery of the upper extremity after stroke. J. rehab. med. 2011;43(6):506-10.

10. Liu W, Mukherjee M, Sun C, Liu H, McPeak LK. Electroacupuncture may help motor recovery in chronic stroke survivors: a pilot study. J. rehabil. res. dev. 2008;45(4):587-95.

11. Wang BH, Lin CL, Li TM, Lin SD, Lin JG, Chou LW. Selection of acupoints for managing upperextremity spasticity in chronic stroke patients. Clin. interv. aging. 2014;9:147-56.

12. Hara Y, Ogawa S, Muraoka Y. Hybrid powerassisted functional electrical stimulation to improve hemiparetic upper-extremity function. Am. j. phys. med. rehabil. 2006;85(12):977-85.
13. Malhotra S, Rosewilliam S, Hermens H, Roffe C, Jones P, Pandyan AD. A randomized controlled trial of surface neuromuscular electrical stimulation applied early after acute stroke: effects on wrist pain, spasticity and contractures. Clin. rehabil. 2013; 27(7):579-90.

14. Mukherjee M, McPeak LK, Redford JB, Sun C, Liu W. The effect of electroacupuncture on spasticity of the wrist joint in chronic stroke survivors. Arch. phys. med. rehab. 2007;88(2):159-66.

15. Cauraugh J, Light K, Kim S, Thigpen M, Behrman A. Chronic motor dysfunction after stroke: recovering wrist and finger extension by electromyography-triggered neuromuscular stimulation. Stroke. 2000;31(6):1360-4.

16. Peurala SH, Pitkänen K, Sivenius J, Tarkka IM. Cutaneous electrical stimulation may enhance sensorimotor recovery in chronic stroke. Clin Rehabil. 2002 Nov;16(7):709-16.

17. Kimberley TJ, Lewis SM, Averbach EJ, Dorsey LL, Lojovich JM, Carey JR. et al. Electrical stimulation driving neuromuscular improvements and cortical in patients with stroke. Exp. brain. res. 2004; 154: 450-460.

18. McDonnell M, Hillier S, Miles T, Thompson PD, Ridding MC. Influence of combined afferent stimulation and task-specific training following stroke: a pilot randomized controlled trial. Neurorehab. neural. repair. 2007;12:435-442.

19. Daly J, Roenigk K, Holcomb J, Rogers JM, Butler K, Gansen J, et al. A randomized controlled trial of functional neuromuscular stimulation in chronic stroke subject. Stroke 2006; 37:172-178.

2O. Fleuren JF, Voerman GE, Erren-Wolters CV, Snoek GJ, Rietman JS, Hermens HJ, Nene AV. Stop using the Ashworth Scale for the assessment of spasticity. J. neurol., neurosurg. psychiatry. 2010;81(1):46-52. 